\title{
AN EXPERIMENTAL EVALUATION ON AIR CONDITIONING PERFORMANCE WITH VAIRTEX AIR DIRECTOR APPLIED ON RESIDENTIAL CONDENSER UNIT
}

\author{
Vien Nguyen and Edgar Matida \\ Department of Mechanical and Aerospace Engineering \\ Carleton University \\ Ottawa, Canada
}

\author{
Gerard Godbout \\ VairTEX Canada Inc. \\ Ottawa, Canada
}

\begin{abstract}
Thermodynamic performance measurements of a 1.5 ton residential air conditioning system and fluid flow hotwire velocity measurements at the outlet of the condenser were performed with and without an add-on device (VairTEX Air Director added on top of the condenser unit). The Air Director modified all three velocity components at the condenser exit. Data from hot-wire confirmed the Air Director reduced the radial component while enhancing swirling factor and adding $48.7 \%$ volumetric flow rate across the condenser unit. By streamlining the flow, the Air Director led to an improvement of 5\% in terms of overall coefficient of performance of the air conditioning unit and $4.5 \%$ reduction in compressor power consumption.
\end{abstract}

Keywords - air conditioning; condenser; coefficient of performance; hot-wire; compressor power; air director; VairTEX

\section{INTRODUCTION}

Typically, residential condensers are placed on a concrete slab outside the house. Due to the simplistic design of the commonly used axial fans in these condenser units (a compromise between overall costs, robustness, and performance), the air flow does not help to extract the heat from the condenser coil effectively, thus increasing power consumption of the compressor. In addition, temperature of incoming air to those coils of a condenser could be influenced by the heated flow escaping from the condenser outlet due to its exit angles [1]. A study by Khankari (2016) also indicated that with an increase in crosswind, the hot plume discharging from the condenser exit bent downward and almost reached the ground in case of higher wind speed [2]. Those factors stated above directly affect the overall performance of the air conditioning (AC) system.

Recently, the improvement of AC performance by investigating the shading effects on the condenser has been performed. Donovan and Butry (2009) estimated savings of $5.2 \%$ in electricity consumption in summertime when trees were planted on the west and south sides of 460 single-houses in Sacramento, CA [3]. Improving air flow across the condenser could help lower power consumption, thus increase the coefficient of performance (COP) of the system. Elsayed and Hariri (2011) suggested in their study that a $10 \%$ reduction in compressor power consumption was achieved by increasing the condenser air flow of about 50\% in split-type air conditioners [4]. A different approach was presented by Danny S. Parker et al. (2005) in which true-airfoil flat fan blades were embedded in a conical diffuser, situated on top of the condenser coils [5]. Parker's attempt successfully reduced noise level of the system while increased air flow by 5\%, and also reduced fan power consumption by $26 \%$. Parker also reported an overall increase on Energy Efficiency Ratio (EER) and COP of approximately 2 $-4 \%$.

The Air Director (AD, VairTEX Canada Inc.) is a passive energy reduction device which can be added to the top of the axial fans of residential condensers. By redirecting the flow upwards, minimizing recirculation, the overall performance of the unit can be improved and at the same time reduce power consumption of the condenser unit.

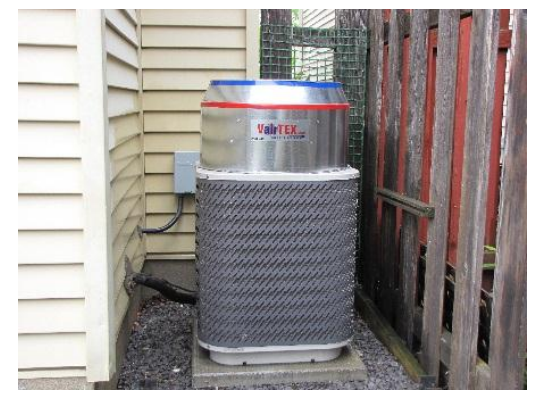

Figure 1. Typical installation of a VairTEX Air Director on top of residential condenser unit

As illustrated in Fig. 1, a VairTEX unit was placed on top of residential condenser. The Air Director [6] consists of three main components, as shown in Fig. 2:

1. Cylindrical body: this component is used to direct the air flow exiting the condenser. The tube's diameter has the same dimension as the condenser exit.

2. Fixed blades: a series of six fixed blades is attached to the interior of a tube in order to promote swirling. In this study, the blades are fixed at $75^{\circ}$ from horizontal plane. It should be noted that as blades are only joined in pairs (i.e. 1 and 6,2 and 5, 3 and 4 ), this leaves 2 small gaps in the center of the device. 
3. Top cowling: is designed to reduce diameter of the cylindrical tube, hence increasing the exit flow velocity.

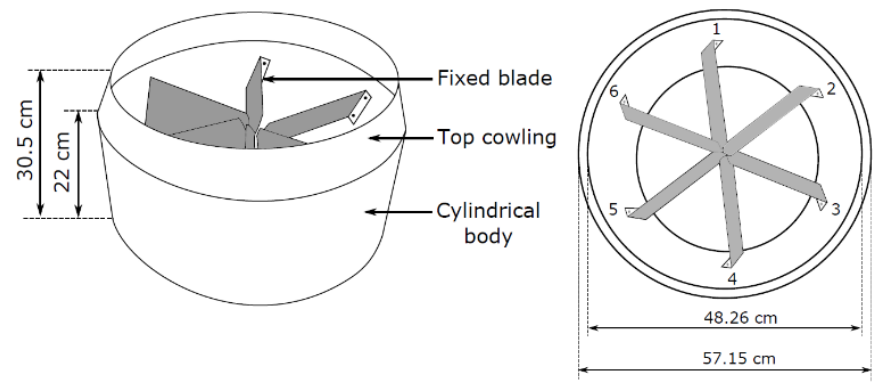

Figure 2. Schematic of the VairTEX Air Director

In the present work, a residential $\mathrm{AC}$ unit, which has been placed and instrumented in a laboratory setting, was used. The thermodynamic performance of the unit was measured with and without the Air Director to assess the overall performance enhancement. In addition, velocity measurement using a hotwire probe was performed to explain the differences in thermodynamic performance. Optimization of the $\mathrm{AD}$ and assessment of cross-wind condition on the AC unit performance were deemed beyond the scope of the current investigation but will be explored in future work.

\section{METHODOLOGY}

\section{A. Thermodynamics Performance Measurement}

\section{1) Experimental Set-up}

A 1.5 ton residential Carrier air conditioning unit was equipped with temperature, pressure and power measurement instruments. The vapor-compression refrigeration cycle in this system uses R-22 as its refrigerant. Being attached to the wall, the evaporator unit was installed above the condenser unit. The condenser, on the other hand, was mounted on the floor where its fan center of rotation is $1.24 \mathrm{~m}$ away from that wall. Also there was an open space around both evaporator and condenser units with a floor to ceiling height of $3.7 \mathrm{~m}$. The refrigerant passed through the compressor where a Wavetek DM7 power meter was calibrated in V-DC scale to measure power consumed by the compressor in $\mathrm{kW}$. Then the refrigerant moved along the condenser coils before reaching the first Ashcroft 2071A Contractor pressure gauges $(0-600 \mathrm{psi})$. A V-groove flow meter type from Kobold Instrument Inc. was installed after the condenser pressure gauge to adjust refrigerant flow rate. The refrigerant then passed through the throttle valve where pressure dropped nearly isenthalpically. Low temperature refrigerant exchanged heat with ambient air across the evaporator coils. The refrigerant pressure after the evaporator was measured by the same type of gauge installed after the condenser. Refrigerant temperatures were measured using type $\mathrm{J}$ thermocouples at five locations, labelled from 1 to 5 while air temperatures were measured at locations 6 and 7, also using type $J$ thermocouples. Schematic of the set-up is illustrated in Fig. 3. The data collected from these instruments were used in the performance analysis for two study cases: with and without the VairTEX Air Director at two different flow rate readings of 0.25 and $0.35 \mathrm{gal} / \mathrm{min}$, respectively. At each flow rate, three repeats were performed for each study case.

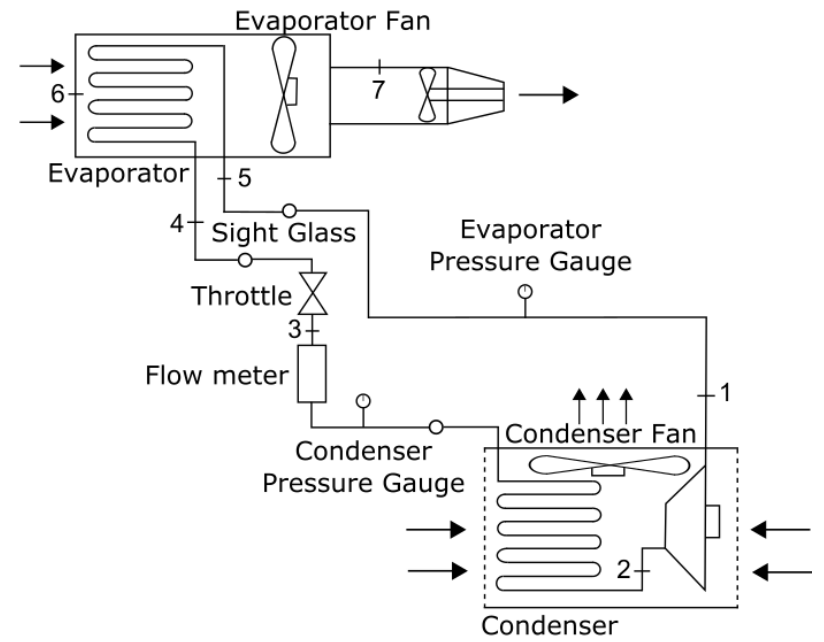

Figure 3. Schematic of the experimental set-up to conduct thermodynamics performance measurement

\section{2) Data Reduction}

Measuring locations are labelled in Fig. 3. By applying the first law of thermodynamics, refrigeration capacity and COP are examined in order to compare the system performance between operating conditions with and without the VairTEX AD. The refrigeration capacity (from the evaporator, or labels 4 to 5 in Fig. 3), denoted as $\dot{Q}_{\text {in }}$, is calculated as

$$
\dot{Q}_{i n}=\dot{m}_{R 22}\left(h_{5}-h_{4}\right)
$$

The COP is defined as the ratio between the obtained cooling over the total power input (taken here as the compressor power, from label 1 to 2 in Fig. 3), hence

$$
C O P=\frac{\dot{m}_{R 22}\left(h_{5}-h_{4}\right)}{\dot{W}_{C}}
$$

\section{B. Velocity Measurement}

\section{1) Fundamental Concept}

To obtain three mean velocity components in turbulent flow field using a single, straight hot-wire probe, a special technique - called six-orientation measurement (SOM) technique, derived by C.F. King [7] was explored. For a single location, the hotwire probe was rotated using six different orientations (turned each time by $30^{\circ}$ ) and measurements were performed. The data obtained at each location were in the form of mean and rootmean-square (RMS) voltages. By applying some assumptions regarding the statistical nature of turbulence, one would be able to solve for the three time-mean Cartesian velocity components. Details of the SOM can be found in [7] [8] [9]. Prior to measurement, the hot-wire probe was calibrated at three different directions using an in-house calibration rig [9]. In addition, temperature correction following Hultmark's method [10] was validated in-house and performed for all velocity measurements.

Ontario Centres of Excellence (OCE) 
2) Experimental set-up

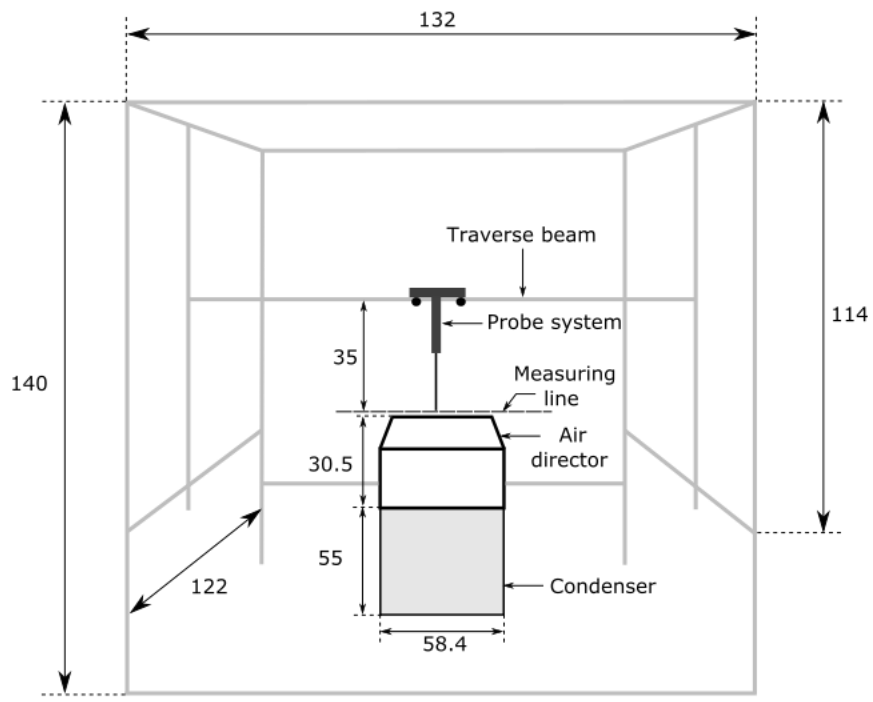

Figure 4. Schematic of a 1D traverse system surrounding the condenser unit. Note that all dimensions are in $\mathrm{cm}$

Fig. 4 provides an illustration of the setup for case with the VairTEX AD added on top of the condenser unit. It should be noted that the measuring line was offset from the exit surface of the air director by a distance of $1 \mathrm{~cm}$. Sitting parallel to the condenser and the AD (Air Director) exit surfaces, the traverse beam was supported by two T-slot beams on both sides. A timing belt was installed along the whole length of the traverse beam. The probe system was fixed on the moving plate which was attached to the belt. This allowed manual translation in one direction for the probe system which consisted of the six-axis rotation device, the probe holder and the hot-wire probe. For the measurement without the $\mathrm{AD}$, the traverse beam was lowered down and distance from the traverse beam to the measuring line, and distance from the measuring line to the condenser exit surface remained the same. 19 measuring locations (radial direction at the mid-plane of the condenser) were taken at the condenser discharge while a total of 11 locations were measured at the $\mathrm{AD}$ top surface.

A single-channel AN-1005 constant temperature anemometry system from A.A Lab Systems Ltd. was used to acquired data from Dantec Dyanmics single, normal hot-wire probe 55P11. The hot-wire was sampled at $4 \mathrm{kHz}$ using DAQ 14-bit NI USB-6009. A sealed junction thermocouple 30-gaugewire $(0.255 \mathrm{~mm})$ type-K with insulation thickness of $0.635 \mathrm{~mm}$ from J-KEM Scientific Inc. was used to perform temperature measurement at the exit surfaces of the condenser and the Air Director. Temperature measurements were recorded simultaneously with measurements from hot-wire for 30 seconds. Readings from the thermocouple were acquired by a portable USB 2.0 full speed thermocouple/voltage input data acquisition module Omega DAQ USB 2401. Data was sampled at $1 \mathrm{kHz}$ which is the maximum rate of the Omega DAQ. All hot-wire and temperature measurements were repeated for three times.

\section{RESULTS AND DISCUSSIONS}

\section{A. Thermodynamic performance}

1) T-s diagram comparison

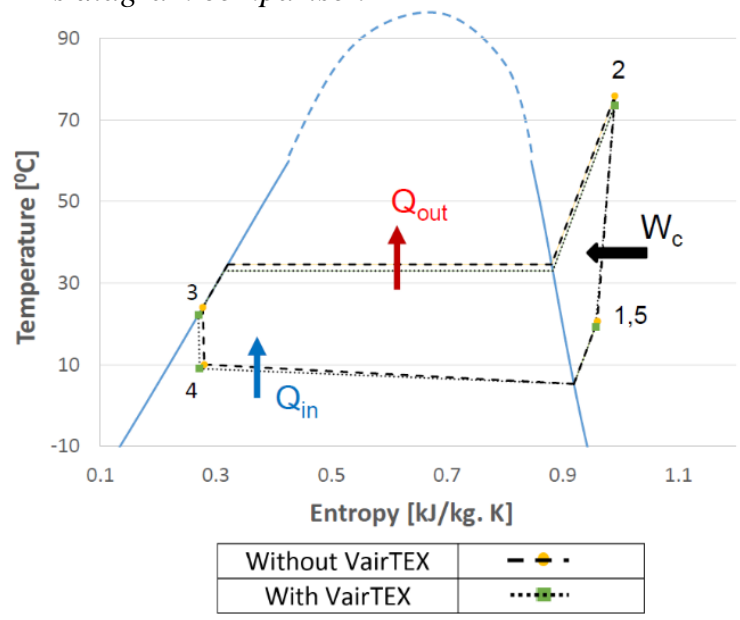

Figure 5. T-s diagram comparison with and without the $A D$ at refrigerant flow rate reading of $0.35 \mathrm{gal} / \mathrm{min}$

Based on mean values recorded at five measuring locations in the experimental setup, the T-s diagram for refrigerant flow rate of $0.35 \mathrm{gal} / \mathrm{min}\left(0.0013 \mathrm{~m}^{3} / \mathrm{min}\right)$ was constructed, as shown in Fig. 5. The blue dash line is an arbitrary line which represents the top of the liquid-vapor dome due to the lack of data above $60^{\circ} \mathrm{C}$. The T-s diagram explains the performance difference between two study cases: with and without the AD (Air Director) from a thermodynamic point of view. Lower pressure during the condensation process (2-3) was recorded in case with the AD. As a consequence, the compressor work (1-2) $\mathrm{W}_{\mathrm{C}}$ was reduced. Moreover, the throttling process in case with the $\mathrm{AD}$ took place at lower temperature, hence adding additional cooling effect to the cycle. From temperature and enthalpy obtained after the throttle (4), calculated pressure at this location indicated that there was a pressure drop during the evaporation process (4-5) for both cases. By reducing the compressor power, a slight increase in refrigeration capacity increases COP, as the AD did in this case. Similarly, the same trend was obtained in case at refrigerant flow rate reading of $0.25 \mathrm{gal} / \mathrm{min}\left(0.0009 \mathrm{~m}^{3} / \mathrm{min}\right)$.

\section{2) Compressor power and $\mathrm{COP}$}

An appreciable reduction in power consumption of $3.7 \%$ and $4.5 \%$ was observed for refrigerant flow rate of 0.25 and 0.35 $\mathrm{gal} / \mathrm{min}$, respectively as the AD was applied on the condenser.

Since power consumed by evaporator and condenser fans stayed constant between study cases, COP depends on two variables: compressor power and refrigeration capacity. As compressor power consumption decreased when the AD was installed while refrigeration capacity remained nearly constant, COP increased by $4 \%$ and $5 \%$ corresponding to flow rate of 0.25 and $0.35 \mathrm{gal} / \mathrm{min}$ when VairTEX AD was installed, as illustrated by Fig. 6. 


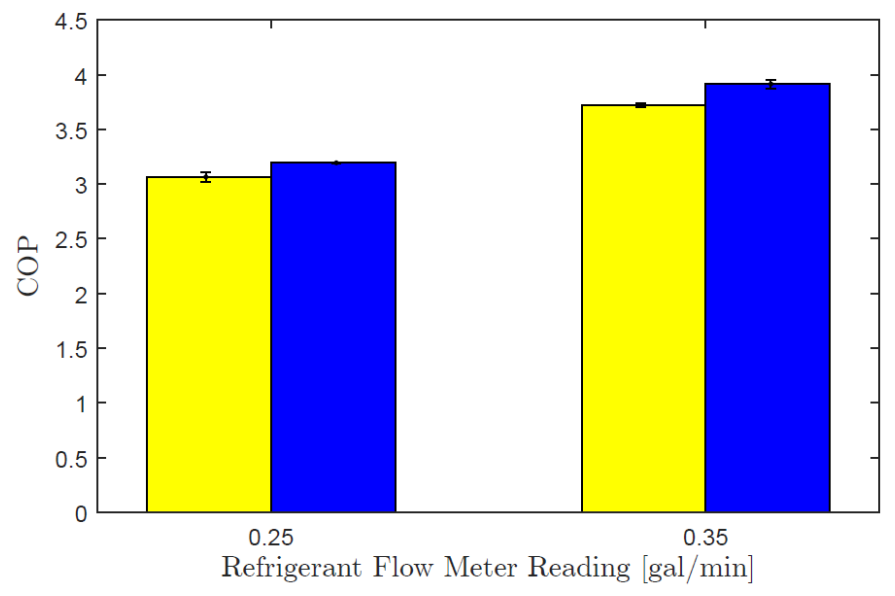

\begin{tabular}{|l}
\hline $\mathrm{W} / \mathrm{O}$ VairTEX $\square \mathrm{W} /$ VairTEX \\
\hline
\end{tabular}

Figure 6. COP comparison between with and without the $\mathrm{AD}$ for two refrigerant flow rate readings

\section{B. Velocity Measurement}

\section{1) Temperature Componenet}

As shown in Fig. 7, it can be seen in case without the AD, the hot air discharged from the condenser resulted in two isolated plumes spanning from $0.1 \mathrm{~m}$ to $0.45 \mathrm{~m}$ of each side. While in case with the $\mathrm{AD}$, the hot discharged was combined into a single plume. Both temperature profiles exhibited asymmetrical caused by heat dissipated from the compressor located inside the condenser.

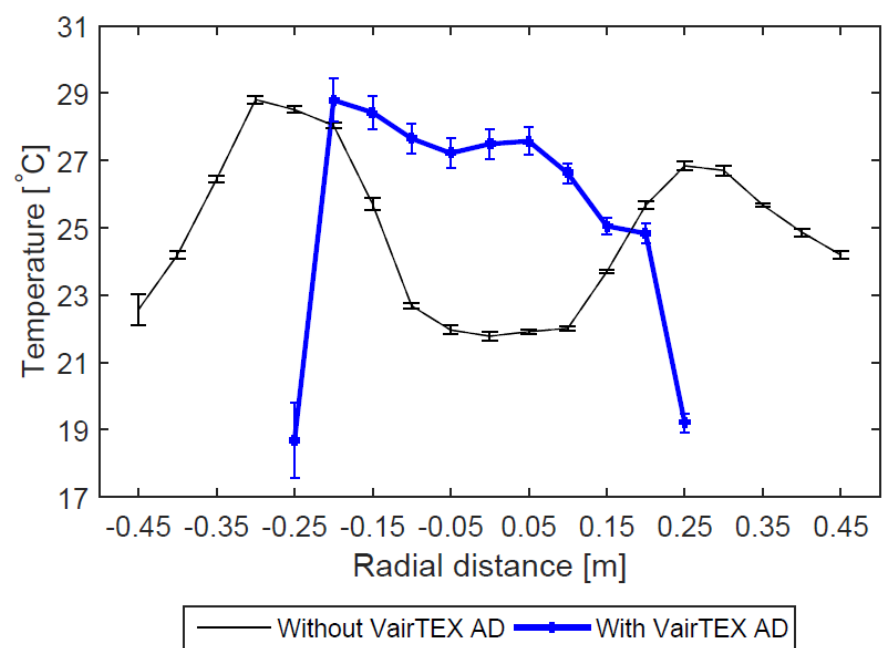

Figure 7. Temperature measured at the condenser surface discharge and at the VairTEX AD exit top

Temperature tremendously increased across the exit of the $\mathrm{AD}$ where an average of $27.1^{\circ} \mathrm{C}$ was obtained in this case, compared to an average of $24.4^{\circ} \mathrm{C}$ without the $\mathrm{AD}$. Temperature measurement has confirmed an increase in heat rejection from the condenser which contributed in an improvement of the condenser unit as a whole.

\section{2) Radial Component}

As displayed in Fig. 8, mean radial component measured for 19 locations at the condenser exit is shown by black line while blue line represents measurements for 11 locations at the AD outlet. First, both radial components with and without the AD are subjected to a non-symmetrical profile due to the imperfection of fan blades and the arrangement of the safety grill with respect to the measuring line at the condenser exit.

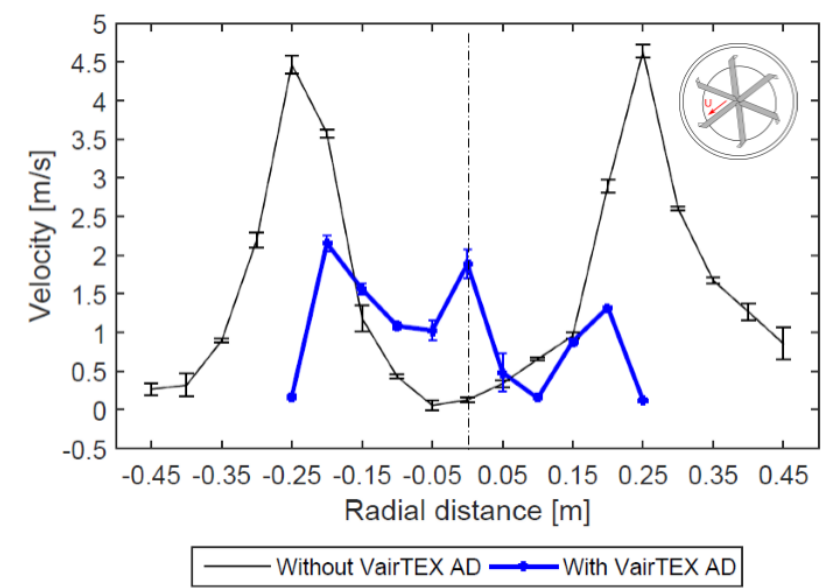

Figure 8. Radial velocity component measured at condenser surface discharge and at the AD top surface

Due to blockage of the motor plate from $-0.1 \mathrm{~m}$ to $0.1 \mathrm{~m}$, low velocities at the center region were recorded in case without the $\mathrm{AD}$, as opposed to high velocities recorded outside the motor plate. When applying at the condenser exit, the $\mathrm{AD}$ reduces radial component significantly. In particular, the peak velocities are reduced to a maximum of $2.2 \mathrm{~m} / \mathrm{s}$ which is more than half of the peak values found in without AD case. At locations where the motor plate exits, radial velocities emerged in this case caused by the blade effect from the AD. Due to the blade angles of the fan and the $\mathrm{AD}$, their interactions could not cancel the radial components completely. Hence, the exit flow is slightly oblique and skewed with strong flow on the left side. Moreover, as mentioned previously, there existed two gaps of $1.5 \mathrm{~cm}$ between three blade joints. The peak appearing at the center of the $\mathrm{AD}$ is induced by the air rushing through those gaps.

\section{3) Tangential Component}

As illustrated in Fig. 9, in the case without the AD, tangential flow pattern follows a similar trend to radial and axial profiles shown above. No actual flow was recorded at stagnation region due to the presence of a motor plate. Values obtained in this region were caused solely by the convection effect from the hotwire sensor.

It is intriguing to examine the tangential component in the case with the $\mathrm{AD}$ where its profile resembles tangential profiles of cyclone devices found in literature [11] [12]. While tangential velocity increases with the increase of radius and after reaching its peak velocity decreases sharply, its value almost equals zero at the two edges and in the center flow field. This indicated that the $\mathrm{AD}$ has added additional swirling to the exit flow. 


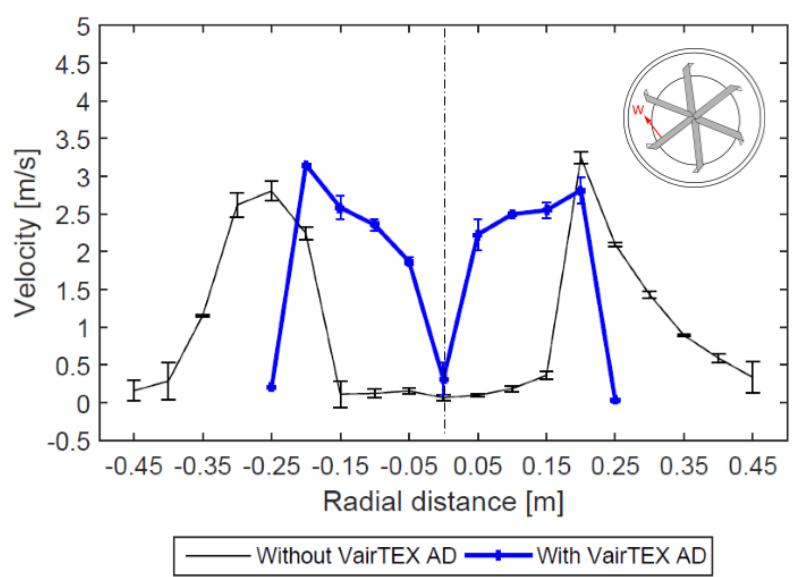

Figure 9. Tangential velocity profile obtained at condenser surface discharge and at the $\mathrm{AD}$ top surface

\section{4) Axial Component}

Double-peak feature and asymmetrical characteristics are observed for both cases, as shown in Fig. 10. However, the AD reduces the difference between two peak values compared to without the AD. The most noticeable improvement of axial component appears at the center region where the motor plate is located. When the $\mathrm{AD}$ is installed the axial flow is significantly increased at the center. As exit flow from the fan was forced to escape through the cylindrical body of the $\mathrm{AD}$, a sufficient height between the condenser exit and the AD blades allow the flow to develop at the center.

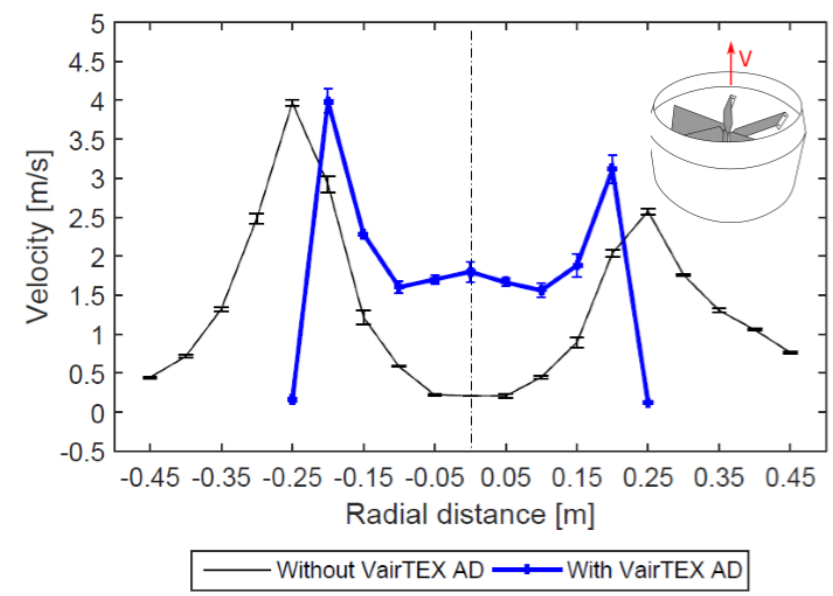

Figure 10. Axial velocity profile obtained at condenser surface discharge and at the $\mathrm{AD}$ top surface

It should be noted that the condenser has the outer diameter of $0.58 \mathrm{~m}$, however the top discharge surface has a diameter of $0.47 \mathrm{~m}$. The volumetric flow rate based on velocity measurement will be evaluated with respect to the top discharge diameter only. By applying the trapezoid integration rule for two outlets: condenser and $\mathrm{AD}$, the volumetric flow rate $\mathrm{Q}$ can be approximated based on the axial velocity profile. A pronounced increment in volumetric flow rate of $48.7 \%$ was recorded when $\mathrm{AD}$ was installed. An increase in axial flow explains why the enhancement of heat rejection at the condenser demonstrated in Fig. 7. As air flow increases, condensing temperature drops, thus compressor power consumption is reduced.

\section{CONCLUSION}

An experimental study was carried out to evaluate the effectiveness of the VairTEX Air Director device on a modified 1.5-ton air conditioner. The experiment was divided into two parts. The first part included thermodynamic performance evaluations at two refrigerant flow rates. As the VairTEX Air Director was installed on the condenser unit, $\mathrm{COP}$ was improved by an appreciable amount of maximum $5 \%$ while the compressor power consumption was reduced by $4.5 \%$ (mainly due better heat transfer, leading to a reduction in refrigerant pressure or needed compressing power at the condenser). Three directional velocity measurements using six-orientation hot-wire technique was employed to characterize air flow discharge from the condenser with and without the device. The radial component was significantly reduced while axial flow was greatly improved, hence increased the volume flow rate by $48.7 \%$. Tangential component at the Air Director exit resembled the profile found in cyclone devices. Heat transfer between ambient and refrigerant at the condenser also increased. Thus, the overall performance of the AC system was improved.

\section{ACKNOWLEDGMENT}

The authors would like to thank Ontario Centres of Excellence (OCE) for providing the Voucher for Innovation and Productivity (VIP) I grant.

\section{REFERENCES}

[1] VairTEX Canada Inc., "Vairtex technical description," Ottawa, 2017.

[2] K. Khankari, "Optimum placement for air-cooled chillers," ASHRAE, vol. 58, 2016.

[3] G. H. Donovan and D. T. Butry, "The value of shade: Estimating the effect of urban trees on summertime electricity use," Energy and Buildings, vol. 41, pp. 662-668, 2009.

[4] A. O. Elsayed and A. S. Hariri, "Effect of condenser air flow on the performance of split air conditioner," in World Renewable Energy Congress, Linkoping, Sweden, 2011.

[5] D. S. Parker, J. R. Sherwin and B. Hibbs, "Development of high efficiency air conditioner condenser fans," in ASHRAE Transactions, Florida, 2005.

[6] G. A. Godbout and D. A. C. Godbout, "Air conditioner turbulence creating energy saver". United States of America Patent 8935937, 20 January 2015.

[7] C. F. King, Some studies of vortex devices - Vortex amplifier performance behavior, $\mathrm{PhD}$ thesis, University College of Wales, 1978.

[8] S. I. Janjua, Turbulence measurements in a complex flowfield using a six-orientation hot-wire probe technique, Master's thesis, Oklahoma State University, 1981.

[9] V. Nguyen, An experimental evaluation on air conditioning performance with VairTEX Air Director applied on residential condenser unit, Master's thesis, Carleton University, 2018.

[10] M. Hultmark and A. J. Smits, "Temperature corrections for constant temperature and constant current hot-wire anemometers," Measurement Science and Technology, 2010.

[11] F. Kaya, I. Karagoz and A. Avci, "Effects of surface roughness on the performance of tangential inlet cyclone separators," Aerosol Science and Technology, vol. 45, pp. 988-995, 2011.

[12] J. Cernecky and K. Plandorova, "The effect of the introduction of an exit tube on the separation efficiency in a cyclone," Brazilian of Chemical Engineering, vol. 30, pp. 627-641, 2013. 